\title{
The NYU Children's Health and Environment Study
}

\author{
Leonardo Trasande ${ }^{1,2,3,4,5}$ - Akhgar Ghassabian ${ }^{1,2,3} \cdot$ Linda G. Kahn $^{1} \cdot$ Melanie H. Jacobson ${ }^{1} \cdot$ Yelena Afanasyeva ${ }^{1,3}$. \\ Mengling Liu ${ }^{2,3} \cdot \mathrm{Yu}_{\mathrm{Chen}}{ }^{2,3}$. Mrudula Naidu ${ }^{1,2}$. Garry Alcedo ${ }^{1}$ - Joseph Gilbert ${ }^{1}$ - Tony T. Koshy ${ }^{1}$ on behalf of the \\ NYU Children's Health and Environment Study Team
}

Received: 9 August 2019 / Accepted: 11 March 2020 / Published online: 25 March 2020

(c) The Author(s) 2020

\begin{abstract}
The aims of the NYU Children's Health and Environment Study (CHES) are to evaluate influences of prenatal non-persistent chemical exposures on fetal and postnatal growth and pool our data with the US National Institutes of Health Environmental influences on Child Health Outcomes (ECHO) Program to answer collaborative research questions on the impact of the preconceptual, prenatal, and postnatal environment on childhood obesity, neurodevelopment, pre/peri/postnatal outcomes, upper and lower airway outcomes, and positive health. Eligible women were $\geq 18$ years old, $<18$ weeks pregnant, had a pregnancy that is not medically threatened, and planned to deliver at NYU Langone Hospital-Manhattan, Bellevue Hospital, or NYU Langone Hospital-Brooklyn. Between March 22, 2016 and April 15, 2019, we recruited 2469 pregnant women, from whom 2193 completed an initial questionnaire and continued into NYU CHES. Of the 2193, 88 miscarried, 28 terminated, and 20 experienced stillbirth, while 57 were lost to follow up. We report here demographic and other characteristics of the 2000 live deliveries (2037 children), from whom 1624 (80\%) consented to postnatal follow-up. Data collection in pregnancy was nested in clinical care, with questionnaire and specimen collection conducted during routine prenatal visits at $<18,18-25$, and $>25$ weeks gestation. These have been followed by questionnaire and specimen collection at birth and regular postpartum intervals.
\end{abstract}

Keywords Endocrine disrupting chemicals $\cdot$ Obesity $\cdot$ Fetal growth $\cdot$ Epigenetics $\cdot$ Metabolomics

\section{Introduction}

The unique vulnerability of children to environmental hazards has been documented in many scientific studies and government reports, including a landmark 1993 US National Academy of Sciences report on pesticide exposures [1]. Yet, studies of children's health have failed to account fully for the range of environmental influences in pregnancy, and the postnatal period that can substantially influence health from

Leonardo Trasande

Leonardo.Trasande@nyumc.org

1 Department of Pediatrics, New York University School of Medicine, New York, NY, USA

2 Department of Environmental Medicine, New York University School of Medicine, New York, NY, USA

3 Department of Population Health, New York University School of Medicine, New York, NY, USA

4 NYU Wagner School of Public Service, New York, NY, USA

5 NYU College of Global Public Health, New York, NY, USA childhood through adult life. The Developmental Origins of Health and Disease hypothesis was first formulated by Barker and colleagues in the context of nutritional influences [2-5]. Nonetheless, it is widely accepted that biological, psychosocial, chemical, and physical exposures are equally influential [6].

Until now, progress toward elucidating the role of the environment in childhood obesity and other chronic conditions has been slow and incremental. Most studies have examined relatively small populations of children [6]; have considered only one chemical exposure at a time; have had little statistical power to examine interactions among chemical, social, and behavioral factors; and have had limited ability to examine gene-environment interactions [7]. Little is known about possible interactions and synergies among chemicals or between chemicals and other environmental hazards, even though the environment of a child includes mixtures of chemical and biological toxicants. Gene-environment interactions and epigenomic effects of exposures are just beginning to be explored. 
For example, the recent explosive increase in the prevalence of obesity reflects a complex interplay among (1) changes in individual behaviors; (2) changes in community structure, lifestyle, and the "built environment"; and (3) exposures to certain synthetic chemicals (e.g., endocrine disruptors) that might disrupt energy balance [6]. Control of the obesity epidemic will require understanding each of these factors and the interplay among them. While previous cohort studies have contributed greatly to identifying many individual-level factors that contribute to the development of obesity in children and its persistence into adulthood in the US and other countries [8-21], these studies have several limitations [6]:

- Previous studies have not fully capitalized on the lifecourse approach to chronic disease epidemiology [22].

- Although some studies have collected genetic data on participants and been able to identify polymorphisms that increase the risk of obesity, they have not simultaneously collected the data on environmental exposures needed to carefully examine the interactions of genetic and environmental factors with diet, physical activity, or epigenetic changes - all of which might predict risk of obesity.

- Many prior cohorts have been limited in their capacities to identify risk factors for obesity that may be unique among Hispanics, a population for which obesity prevalence is increasing especially rapidly [23, 24].

- Past studies have not assessed features of the built environment that encourage healthy diet and physical activity among children living in urban areas [25].

In the context of the broad array of environmental stressors, increasing human and laboratory evidence suggests that exogenous chemicals influence developmental metabolic programming and provoke oxidative stress, a major pathophysiologic mechanism that underlies cardiometabolic risks [26]. These include (1) phthalates (used to soften plastics and as scents), which increase expression of peroxisome proliferator-activated receptors [27] that play key roles in lipid and carbohydrate metabolism [28]; (2) bisphenols (found in aluminum can linings and thermal paper receipts), which are mildly estrogenic, increase fat in adipocytes [29], and disrupt pancreatic $\beta$-cell function; (3) polycyclic aromatic hydrocarbons (PAHs, present in air pollution), which promote inflammation and increase visceral fat in animal models [30, 31]; and (4) organophosphate pesticides (OPs), which are thyroid hormone antagonists that contribute to pre-diabetes, lipid metabolism abnormalities, and obesity in animals [32].

Longitudinal studies of prenatal exposure in humans, especially for phthalates and bisphenols, have not yielded expected findings [33-37]. This might be attributed to (1) limited exposure assessment via collection of a limited number of spot samples during later pregnancy, which restricts insight into how the effects of exposure depend on the earliest stages of fetal development; (2) lack of fetal growth data to evaluate intrauterine effects; (3) use of body mass index (BMI) rather than specific measures of fat mass; (4) failure to measure replacements of bisphenol A and di2-ethylhexylphthalate, or DEHP (particularly bisphenol S, or BPS, diisononylphthalate, or DINP, and diisodecylphthalate, or DIDP) which have begun to be used over the past decade; and (5) lack of mechanistic insight.

The purpose of this manuscript is to describe the NYU Children's Health and Environment Study (CHES), which was designed to overcome these limitations and identify environmental and genetic causes of normal and abnormal growth, development, and health from fetal life onward.

\section{Scope of research}

The general aims of NYU CHES are to:

- Evaluate influences of prenatal non-persistent chemical exposures on fetal and postnatal growth.

- Evaluate prenatal non-persistent chemical exposures in relation to epigenetic marks and gene expression.

- Identify metabotypes related both to exposures and cardiometabolic outcomes, and assess exposures and metabotypes in relation to oxidative stress and perturbations of adiponectin, leptin, and sex hormones.

- Pool our data with that of other cohorts in the US National Institutes of Health (NIH) Environmental influences on Child Health Outcomes (ECHO) program and answer collaborative research questions on the impact of the preconceptual, prenatal, and postnatal environment on childhood obesity, neurodevelopment, pre/peri/postnatal outcomes, upper and lower airway outcomes, and positive health.

\section{Study population and design}

\section{Overview}

NYU CHES is a clinically enrolled, prospective cohort study from fetal life onward. Since March 2016, NYU CHES staff enrolled pregnant women into a biobank study from three NYU Grossman School of Medicine affiliates: NYU Langone Hospital-Manhattan, Bellevue Hospital, and NYU Langone Hospital—Brooklyn, diverse hospitals serving a wide array of populations. Formerly known as Tisch Hospital, NYU Langone Hospital-Manhattan is a major acute care center for the New York City metropolitan area. Bellevue is the flagship hospital of the largest municipal hospital 
system in North America (the New York City Health and Hospitals Corporation). NYU Langone Hospital-Brooklyn's Family Health Center is the second largest federally qualified health center in the nation.

\section{Eligibility and enrollment}

Eligible women were $\geq 18$ years old, $<18$ weeks pregnant, had a pregnancy that was not medically threatened, and planned to deliver at one of the study hospitals. Study staff were bilingual and study materials were available in English, Spanish, and Chinese. To proceed from the biobank study into NYU CHES, participants must have completed an initial questionnaire collecting sociodemographic data and medical history, as well as behaviors, exposures, and experiences in the first trimester.

\section{Study cohort}

\section{Pregnant women and their children}

Between March 22, 2016 and April 15, 2019, we enrolled 2469 pregnant women into a pregnancy biobank, of whom 2193 women completed a questionnaire and continued into NYU CHES (Fig. 1). From 276 pregnant participating in the
Fig. 1 NYU Children's Health and Environment Study (NYU CHES): first 2000 births
Enrolled in pregnancy biobank

$$
2,469
$$

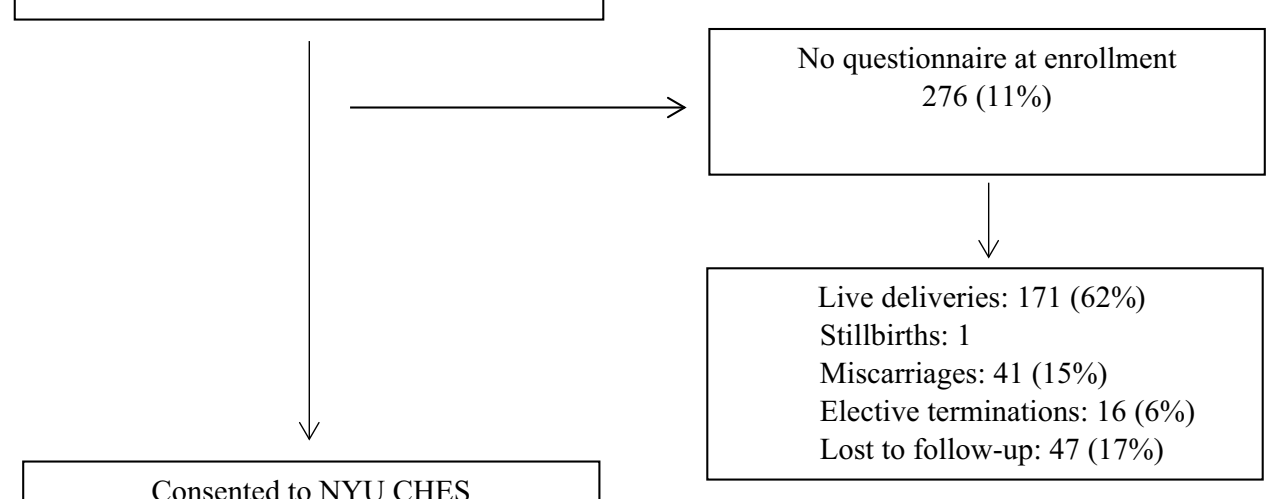

2,193

Stillbirths: $20(1 \%)$

Miscarriages: 88 (4\%)

Elective terminations: $28(1 \%)$

Lost to follow-up: 57 (3\%)
Live deliveries

2,000 ( $91 \%$ of NYU CHES participants)

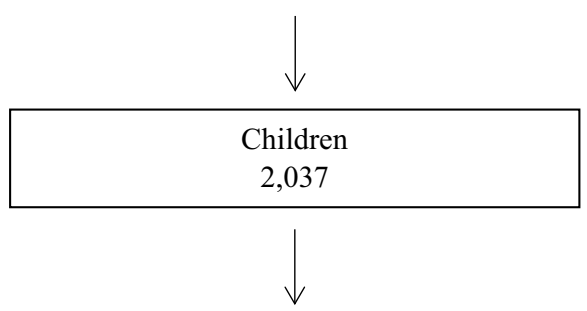

Children whose mothers consented to postnatal phase, including data sharing with the NIH Environmental influences on Child Health Outcomes (ECHO) Program $1,624(80 \%)$ 
biobank, who were not enrolled in NYU CHES because of no questionnaire at enrollment (despite all our efforts), we are aware of 171 live births, while 16 electively terminated and 1 had a stillbirth. Early miscarriage may have contributed to the lack of a questionnaire in 41 of the 276 . These represent an important population to study together with miscarriages in women who did complete a questionnaire ( $n=88$, see below), as environmental exposures in early pregnancy may have differential effects on earlier as opposed to later miscarriage among those who completed questionnaires. Of 2193 pregnant women who continued into NYU CHES, 88 miscarried (mentioned above), 28 terminated, and 20 experienced stillbirth, while 57 were lost to follow up. The 2000 live deliveries resulted in 2037 children; the mothers of $1624(80 \%)$ of these children consented to join the postnatal phase of the study and gave permission to share their children's identifiable data with the ECHO program.

The cohort is multiracial, multiethnic, and has substantial socioeconomic diversity (Table 1). The NYU LangoneManhattan mothers are older, less likely to be Hispanic, more likely to have private health insurance, more likely to be employed, and more likely to be married or partnered; have higher income and education; and have lower BMI than the participants from the other two study sites. Across all recruitment locations, the vast majority are married or partnered and never smoked. While Manhattan mothers were more likely to have used alcohol prior to pregnancy $(20 \%)$, $10 \%$ of participants from two other recruitment locations continued to use alcohol during pregnancy.

To evaluate whether study participants are representative of their respective hospital populations, we compared their aggregate demographic characteristics to those of all patients seen during the same time period at NYU Langone-Manhattan and Brooklyn, the two recruitment locations for which we had hospital-wide information (Table 2). Data for all patients who had their first prenatal visit at these two locations between March 22, 2016 and April 15, 2019 and met the study eligibility criteria were aggregated. Because we did not approach every patient who received prenatal care at these sites, the percentage of study participants should not be misinterpreted as a participation rate. Descriptive data suggest that maternal age, race and ethnicity, insurance status, and parity were nearly identical among participants compared to the overall clinic populations. Participants appeared to be more likely to be married, however, compared to the overall population of pregnant women who visited these clinical sites.

To compare NYU CHES births with those in all of New York City, we obtained aggregate-level data on women who gave birth in New York City in 2016 and who sought prenatal care in the first trimester using publicly available data from the Bureau of Vital Statistics at the New York City Department of Health and Mental Hygiene [38].
Comparison of NYU CHES live births to the population of births in New York City reveals substantial similarity, supporting the broader generalizability of findings from NYU CHES (Table 3). Although NYU CHES participants with live births are substantially more likely to be Hispanic, less likely to be Asian or Non-Hispanic Black, and more likely to be married than those who gave birth across New York City, distributions of maternal age, education, parity, and pre-pregnancy BMI category are similar, as are their children's delivery method, gestational age at birth, sex, and birth weight.

From 2193 pregnant participating in NYU CHES, 20 women had stillbirths, 28 had elective terminations, and 88 experienced miscarriages. The cohort includes 37 multiple births (Table 4). Cesarean section rates were comparable at the two NYU Langone hospitals, and higher than at Bellevue (32-37\% vs. 22\%). Preterm birth was higher at Bellevue versus the other sites ( $10 \%$ vs. $7-8 \%$ for preterm birth), in keeping with known associations of sociodemographic factors with preterm birth [39, 40]. Nonetheless, low birth weight was the lowest in NYU Brooklyn (6\%) compared to NYU Manhattan and Bellevue ( 9 and $8 \%$, respectively).

\section{Data collection in the prenatal phase}

Visits in pregnancy were nested in clinical care, with questionnaire and specimen collection conducted during three routine prenatal visits in the following intervals: $<18$ weeks; 18-25 weeks; and $>25$ weeks gestation. These were followed by visits at birth and at regular postpartum intervals (which occur separately from clinical care). Maternal blood, urine, and saliva samples were collected at each prenatal visit, vaginal samples were collected at least once in pregnancy, and fecal samples were collected in a subsample postnatally. At birth, cord blood samples included whole blood, serum, plasma, and PAXgene tubes for RNA analysis. Placental cores $(2 \times 2 \mathrm{~cm})$ and segments of the umbilical cord were also collected. Maternal urine samples were available for all three time points during pregnancy in a majority of participants, and nearly four-fifths of participants had placental and/or cord blood samples available (Table 5).

\section{Questionnaires and medical chart abstraction}

Questionnaires administered in the first two pregnancy intervals and at birth collected information on participant and partner demographics as well as participants' reproductive health and history, medication and substance use, employment, address, and home life (Table 6). The questionnaires included a variety of validated psychosocial scales (the Pregnancy-Related Anxiety Scale [41] and the Patient Health Questionnaire-9 (PHQ-9) [42] for measurement of 
Table 1 Descriptive characteristics of NYU CHES study participants by recruitment location

\begin{tabular}{|c|c|c|c|c|}
\hline Characteristic $^{\mathrm{a}}$ & $\begin{array}{l}\text { NYU Langone } \\
\text { Hospital-Manhattan } \\
\mathrm{n}=1076\end{array}$ & $\begin{array}{l}\text { NYU Langone } \\
\text { Hospital-Brooklyn } \\
\mathrm{n}=673\end{array}$ & $\begin{array}{l}\text { Bellevue Hospital } \\
n=444\end{array}$ & $\begin{array}{l}\text { Total NYU CHES } \\
n=2193\end{array}$ \\
\hline Age at enrollment, mean (SD), years & $33.9(4.5)$ & $29.3(5.8)$ & $30.2(5.9)$ & $31.8(5.6)$ \\
\hline \multicolumn{5}{|l|}{ Race/ethnicity, n (\%) } \\
\hline Hispanic & $169(16 \%)$ & $563(84 \%)$ & $339(77 \%)$ & $1071(49 \%)$ \\
\hline White, not of Hispanic origin & $652(61 \%)$ & $42(6 \%)$ & $33(8 \%)$ & $727(33 \%)$ \\
\hline Black, not of Hispanic origin & $62(6 \%)$ & $33(5 \%)$ & $35(8 \%)$ & $130(6 \%)$ \\
\hline Asian & $143(13 \%)$ & $27(4 \%)$ & $23(5 \%)$ & $193(9 \%)$ \\
\hline Other & $12(1 \%)$ & $4(1 \%)$ & $4(1 \%)$ & $20(1 \%)$ \\
\hline Multiple & $36(3 \%)$ & $2(<1 \%)$ & $6(1 \%)$ & $44(2 \%)$ \\
\hline \multicolumn{5}{|l|}{ Marital status, n (\%) } \\
\hline Married/living with a partner & $1024(95 \%)$ & $543(81 \%)$ & $344(78 \%)$ & $1911(88 \%)$ \\
\hline Divorced/separated & $10(1 \%)$ & $23(3 \%)$ & $15(3 \%)$ & $48(2 \%)$ \\
\hline Single/widowed & $38(4 \%)$ & $104(16 \%)$ & $84(19 \%)$ & $226(10 \%)$ \\
\hline \multicolumn{5}{|l|}{ Education, n (\%) } \\
\hline High school or less & $33(3 \%)$ & $434(66 \%)$ & $237(54 \%)$ & $704(32 \%)$ \\
\hline Some college but no degree & $64(6 \%)$ & $110(17 \%)$ & $82(19 \%)$ & $256(12 \%)$ \\
\hline Associate degree & $31(3 \%)$ & $43(6 \%)$ & $32(7 \%)$ & $106(5 \%)$ \\
\hline Bachelor's degree & $380(36 \%)$ & $55(8 \%)$ & $63(15 \%)$ & $498(23 \%)$ \\
\hline Post graduate degree & $559(52 \%)$ & $20(3 \%)$ & $23(5 \%)$ & $602(28 \%)$ \\
\hline \multicolumn{5}{|l|}{ Household income, n (\%) } \\
\hline Less than $\$ 30,000$ & $24(2 \%)$ & $196(30 \%)$ & $142(34 \%)$ & $362(17 \%)$ \\
\hline$\$ 30,000-\$ 49,999$ & $36(4 \%)$ & $78(12 \%)$ & $64(15 \%)$ & $178(8 \%)$ \\
\hline$\$ 50,000-\$ 74,999$ & $90(8 \%)$ & $31(5 \%)$ & $20(5 \%)$ & $141(7 \%)$ \\
\hline$\$ 75,000-\$ 99,999$ & $93(9 \%)$ & $6(1 \%)$ & $7(2 \%)$ & $106(5 \%)$ \\
\hline$\$ 100,000$ or more & $778(74 \%)$ & $18(3 \%)$ & $9(2 \%)$ & $805(38 \%)$ \\
\hline Don’t know & $34(3 \%)$ & $323(49 \%)$ & $172(42 \%)$ & $529(25 \%)$ \\
\hline Employed, n (\%) & $916(86 \%)$ & $271(41 \%)$ & $225(51 \%)$ & $1412(65 \%)$ \\
\hline \multicolumn{5}{|l|}{ Insurance, $\mathrm{n}(\%)$} \\
\hline Public & $139(13 \%)$ & $587(88 \%)$ & $413(96 \%)$ & $1139(52 \%)$ \\
\hline Private & $934(87 \%)$ & $83(12 \%)$ & $15(4 \%)$ & $1032(48 \%)$ \\
\hline \multicolumn{5}{|l|}{$\begin{array}{l}\text { Use of cigarettes and other related products }{ }^{\mathrm{b}} \text { during } \\
\text { pregnancy, } \mathrm{n}(\%)\end{array}$} \\
\hline Never & $991(92 \%)$ & $607(90 \%)$ & $385(87 \%)$ & $1983(91 \%)$ \\
\hline Used, stopped in pregnancy & $72(7 \%)$ & $57(9 \%)$ & $51(11 \%)$ & $180(8 \%)$ \\
\hline Used, continued in pregnancy & $10(1 \%)$ & $9(1 \%)$ & $8(2 \%)$ & $27(1 \%)$ \\
\hline \multicolumn{5}{|l|}{ Alcohol use during pregnancy, n (\%) } \\
\hline Never & $155(15 \%)$ & $368(55 \%)$ & $211(48 \%)$ & $734(33 \%)$ \\
\hline Used, stopped in pregnancy & $700(65 \%)$ & $234(35 \%)$ & $191(43 \%)$ & $1125(52 \%)$ \\
\hline Used, continued in pregnancy & $215(20 \%)$ & $66(10 \%)$ & $39(9 \%)$ & $320(15 \%)$ \\
\hline Pre-pregnancy body mass index, mean (SD), $\mathrm{kg} / \mathrm{m}^{2}$ & $24.7(5.0)$ & $27.5(5.5)$ & $28.6(6.5)$ & $26.3(5.7)$ \\
\hline Nulliparous, n (\%) & $687(64 \%)$ & $227(34 \%)$ & $203(46 \%)$ & $1117(51 \%)$ \\
\hline
\end{tabular}

${ }^{a}$ Missing data: race/ethnicity: $8(<1 \%)$; marital status: $8(<1 \%)$; education: $27(1 \%)$; household income: $72(3 \%)$; insurance: $22(1 \%)$; cigarettes and other related prodcuts use: $3(<1 \%)$; alcohol use: 14 (1\%); pre-pregnancy body mass index: 27 (1\%); employment: $14(1 \%)$; parity: $6(<1 \%)$ ${ }^{\mathrm{b}}$ Includes cigars, cigarillos, hookah, patches, gums, and other nicotine products

depression). Women were also asked about their sleep during pregnancy using the Pittsburgh Sleep Quality Index (PSQI) [43]. Once during pregnancy, participants completed the Diet History Questionnaire II (DHQ II), a publicly available food frequency questionnaire developed by the US National Cancer Institute [44]. Of the four versions of the DHQ, we used the version that asks about diet in the past year, including portion size (available and validated in 
Table 2 Representativeness of NYU CHES participants from NYU Langone-Manhattan and Brooklyn

\begin{tabular}{|c|c|c|c|c|}
\hline \multirow[t]{3}{*}{ Characteristic } & \multicolumn{2}{|c|}{$\begin{array}{l}\text { NYU Langone Obstetrics \& Gynecology } \\
\text { Associates (New York, NY) }\end{array}$} & \multicolumn{2}{|c|}{$\begin{array}{l}\text { Sunset Park Family Health Center } \\
\text { at NYU Langone, Women's Health } \\
\text { (Brooklyn, NY) }\end{array}$} \\
\hline & $\begin{array}{l}\text { All patients seen in the } \\
\text { clinic }\end{array}$ & $\begin{array}{l}\text { NYU CHES partici- } \\
\text { pants }\end{array}$ & $\begin{array}{l}\text { All patients seen in the } \\
\text { clinic }\end{array}$ & $\begin{array}{l}\text { NYU CHES } \\
\text { participants }\end{array}$ \\
\hline & $\mathrm{n}=8176$ & $\mathrm{n}=1076$ & $\mathrm{n}=2723$ & $\mathrm{n}=673$ \\
\hline Age at first prenatal visit, mean (SD), years & $34.1(5.1)$ & $33.9(4.5)$ & $29.9(6.1)$ & $29.3(5.8)$ \\
\hline \multicolumn{5}{|l|}{ Race/ethnicity, n (\%) } \\
\hline Hispanic & $766(10 \%)$ & $169(16 \%)$ & $1988(74 \%)$ & $563(84 \%)$ \\
\hline White, not of Hispanic origin & $5106(65 \%)$ & $652(61 \%)$ & $223(8 \%)$ & $42(6 \%)$ \\
\hline Black, not of Hispanic origin & $538(7 \%)$ & $62(6 \%)$ & $147(5 \%)$ & $33(5 \%)$ \\
\hline Asian & $972(13 \%)$ & $143(13 \%)$ & $111(4 \%)$ & $27(4 \%)$ \\
\hline Other/multiple & $425(5 \%)$ & $48(4 \%)$ & $237(9 \%)$ & $6(1 \%)$ \\
\hline Missing & 369 & 2 & 17 & 2 \\
\hline \multicolumn{5}{|l|}{ Marital status, n (\%) } \\
\hline Married/living with a partner & $5279(77 \%)$ & $1024(95 \%)$ & $1131(46 \%)$ & $543(81 \%)$ \\
\hline Divorced/separated & $60(1 \%)$ & $10(1 \%)$ & $30(1 \%)$ & $23(3 \%)$ \\
\hline Single & $1451(21 \%)$ & $38(4 \%)$ & $1283(52 \%)$ & $104(16 \%)$ \\
\hline Other & $41(1 \%)$ & 0 & $9(<1 \%)$ & 0 \\
\hline Missing & 1345 & 4 & 270 & 3 \\
\hline Nulliparous, n (\%) & $4939(62 \%)$ & $687(64 \%)$ & $1096(40 \%)$ & $227(34 \%)$ \\
\hline Missing & 167 & 0 & 13 & 2 \\
\hline \multicolumn{5}{|l|}{ Insurance, $\mathrm{n}(\%)$} \\
\hline Public & $1112(14 \%)$ & $139(13 \%)$ & $2366(87 \%)$ & $587(88 \%)$ \\
\hline Private & $6960(86 \%)$ & $934(87 \%)$ & $341(13 \%)$ & $83(12 \%)$ \\
\hline Missing & 104 & 3 & 16 & 3 \\
\hline Pre-pregnancy BMI, mean (SD), kg/m² & $24.8(5.2)$ & $24.7(5.0)$ & $28.1(6.1)$ & $27.5(5.5)$ \\
\hline Missing & 3509 & 3 & 1739 & 15 \\
\hline Gestational age at delivery, mean (SD), weeks ${ }^{\mathrm{a}}$ & $38.8(2.6)$ & $39.0(1.7)$ & $38.9(2.0)$ & $39.0(1.8)$ \\
\hline Missing & 1930 & 23 & 816 & 12 \\
\hline Child sex, male, $\mathrm{n}(\%)^{\mathrm{b}}$ & $3256(51 \%)$ & $534(53 \%)$ & $980(51 \%)$ & $311(51 \%)$ \\
\hline Missing & 40 & 27 & 11 & 11 \\
\hline Birth weight, mean (SD), $\mathrm{g}^{\mathrm{b}}$ & $3215(607)$ & $3226(553)$ & $3052(811)$ & $3279(536)$ \\
\hline Missing & 1859 & 52 & 2534 & 30 \\
\hline
\end{tabular}

${ }^{\mathrm{a}}$ Among live deliveries

${ }^{\mathrm{b}}$ Among live children

English and translated to Spanish). Maternal physical activity was assessed in all three intervals during pregnancy using the International Physical Activity Questionnaire-Short Form (IPAQ) [45].

Substantial data are obtained directly from electronic health records, including maternal age at enrollment, parity/ gravity, gestational hypertension, preeclampsia, weight and blood pressure at each prenatal visit, and mode of delivery. Gestational diabetes will be evaluated using the American College of Obstetrics and Gynecology guidelines for glucose tolerance testing. Fetal growth data and estimated gestational dating collected during regular ultrasounds are also extracted from electronic health records, including crown-rump length, biparietal diameter, head circumference, transverse cerebellar diameter, femur length, and abdominal circumference, as appropriate (Table 6).

\section{Specimen analyses}

With ECHO support, we are analyzing urine at each time point in pregnancy for OP metabolites, phthalate metabolites, bisphenols, PAHs, biomarkers of oxidative stress (F2-isoprostane and 8-hydroxydeoxyguanosine), cotinine, creatinine, and metabolomic indicators (Table 6). We are analyzing adiponectin and leptin in cord blood, as well as cytokines in serum at each pregnancy time point 
Table 3 Comparison of NYU CHES participants with women who delivered live births in New York City, 2016
Characteristic

2016 New York City birth data

NYU CHES participants with live deliveries

$\mathrm{n}=88,924$

$\mathrm{n}=2000$

Mother's
$<20$
$20-24$
$25-29$
$30-34$
$35-39$
$40+$

Race/ethnicity, n (\%)

Hispanic

Asian/Pacific Islander

Non-Hispanic White

Non-Hispanic Black

Other

Missing

Marital status, n (\%)

Married

Not married

Missing

Education, $\mathrm{n}(\%)$

High school or less

Some college, associate degree

Bachelor's degree

Post graduate degree

Missing

Nulliparous, n (\%)

Missing

Pre-pregnancy BMI category, n (\%)

Underweight

Normal

Overweight

Obese

Missing

Multiple births, n (\%)

Delivery method, $\mathrm{n}(\%)$

Vaginal

Cesarean section

Missing

Gestational age category, $\mathrm{n}(\%)$

17-31 weeks

32-33 weeks

34-36 weeks

37-47 weeks

Missing

Preterm births (<37 weeks), n (\%)

Missing

Child sex, male, $\mathrm{n}(\%)^{\mathrm{a}}$

Missing

Birth weight category, $\mathrm{n}(\%)^{\mathrm{a}}$
$1754(2 \%)$

$45(2 \%)$

$11,977(14 \%)$

$217(11 \%)$

$22,442(25 \%)$

$425(21 \%)$

$28,737(32 \%)$

$732(37 \%)$

$18,850(21 \%)$

$454(23 \%)$

$5164(6 \%)$

$127(6 \%)$

$23,614(27 \%)$

$965(48 \%)$

$16,586(18 \%)$

$180(9 \%)$

$33,737(38 \%)$

677 (34\%)

$13,843(16 \%)$

$114(6 \%)$

$1096(1 \%)$

$59(3 \%)$

48

5

$59,466(67 \%)$

$1757(88 \%)$

$29,458(33 \%)$

0

$242(12 \%)$

1

$31,321(35 \%)$

$635(32 \%)$

$327(16 \%)$

457 (23\%)

$564(29 \%)$

17

$1002(50 \%)$

3

4920 (5\%)

$41(2 \%)$

$48,515(55 \%)$

$947(48 \%)$

$571(29 \%)$

$422(21 \%)$

19

$37(2 \%)$

$59,366(67 \%)$

$1264(67 \%)$

$29,558(33 \%)$

$615(33 \%)$

0

121

$1222(1 \%)$

$14(1 \%)$

$912(1 \%)$

$18(1 \%)$

$5686(7 \%)$

$124(6 \%)$

$81,104(91 \%)$

1797 (92\%)

0

47

$7820(9 \%)$

0

$156(8 \%)$

47

$45,423(51 \%)$

0

$1031(52 \%)$

56 
Table 3 (continued)

\begin{tabular}{lll}
\hline Characteristic & 2016 New York City birth data & $\begin{array}{l}\text { NYU CHES par- } \\
\text { ticipants with live } \\
\text { deliveries } \\
\mathrm{n}=2000\end{array}$ \\
\hline$<1000$ & $\mathrm{n}=88,924$ & $6(<1 \%)$ \\
$1000-1499$ & $521(1 \%)$ & $9(<1 \%)$ \\
$1500-1999$ & $649(1 \%)$ & $34(2 \%)$ \\
$2000-2499$ & $1328(1 \%)$ & $101(5 \%)$ \\
$2500-2999$ & $4613(5 \%)$ & $389(20 \%)$ \\
$3000-3499$ & $17,852(20 \%)$ & $777(40 \%)$ \\
$3500-3999$ & $35,977(40 \%)$ & $490(26 \%)$ \\
$4000-4499$ & $22,295(25 \%)$ & $105(5 \%)$ \\
$4500-4999$ & $5027(6 \%)$ & $17(1 \%)$ \\
$5000+$ & $605(1 \%)$ & $1(<1 \%)$ \\
Missing & $57(<1 \%)$ & 108 \\
Low birth weight $(<2500 \mathrm{~g}), \mathrm{n}(\%)^{\mathrm{a}}$ & 0 & $150(8 \%)$ \\
Missing & $7111(8 \%)$ & 108 \\
\hline
\end{tabular}

${ }^{a}$ NYU CHES: among live children, $n=2037$

Table 4 Birth outcomes in the initial phase of NYU CHES

\begin{tabular}{|c|c|c|c|c|}
\hline Characteristic $^{\mathrm{a}}$ & $\begin{array}{l}\text { NYU Langone Hospi- } \\
\text { tal-Manhattan } \\
\mathrm{n}=1076\end{array}$ & $\begin{array}{l}\text { NYU Langone Hospi- } \\
\text { tal-Brooklyn } \\
n=673\end{array}$ & $\begin{array}{l}\text { Bellevue Hospital } \\
n=444\end{array}$ & $\begin{array}{l}\text { Total } \\
\mathrm{n}=2193\end{array}$ \\
\hline \multicolumn{5}{|l|}{ Pregnancy outcome, n (\%) } \\
\hline Live delivery & $1006(95 \%)$ & $611(93 \%)$ & $383(90 \%)$ & $2000(94 \%)$ \\
\hline Stillbirth & $9(1 \%)$ & $7(1 \%)$ & $4(1 \%)$ & $20(1 \%)$ \\
\hline Miscarriage & $28(3 \%)$ & $33(5 \%)$ & $27(6 \%)$ & $88(4 \%)$ \\
\hline Elective termination & $11(1 \%)$ & $5(1 \%)$ & $12(3 \%)$ & $28(1 \%)$ \\
\hline Multiple births (all twins), $\mathrm{n}(\%)^{\mathrm{b}}$ & $24(2 \%)$ & $9(1 \%)$ & $4(1 \%)$ & $37(2 \%)$ \\
\hline Gestational age at delivery, mean (SD), weeks ${ }^{\mathrm{b}}$ & $39.0(1.7)$ & $39.0(1.8)$ & $39.0(2.0)$ & $39.0(1.8)$ \\
\hline Preterm births (<37 weeks), n $(\%)^{\mathrm{b}}$ & $79(8 \%)$ & $40(7 \%)$ & $37(10 \%)$ & $156(8 \%)$ \\
\hline \multicolumn{5}{|l|}{ Delivery method, $\mathrm{n}(\%)^{\mathrm{b}}$} \\
\hline Vaginal & $591(63 \%)$ & $394(68 \%)$ & $279(78 \%)$ & $1264(67 \%)$ \\
\hline Cesarean section & $352(37 \%)$ & $186(32 \%)$ & $77(22 \%)$ & $615(33 \%)$ \\
\hline Child sex, n (\%), male ${ }^{\mathrm{c}}$ & $534(53 \%)$ & $311(51 \%)$ & $186(50 \%)$ & $1031(52 \%)$ \\
\hline Birth weight, mean (SD), $\mathrm{g}^{\mathrm{c}}$ & $3226(553)$ & $3279(536)$ & $3284(571)$ & $3284(568)$ \\
\hline Low birth weight $(<2500 \mathrm{~g}), \mathrm{n}(\%)^{\mathrm{c}}$ & $86(9 \%)$ & $36(6 \%)$ & $28(8 \%)$ & $150(8 \%)$ \\
\hline
\end{tabular}

${ }^{a}$ Missing data: pregnancy outcome: 57 (3\%); gestational age at delivery and preterm births: 47 (2\%), delivery method: 121 (6\%); child sex: 56 (3\%); birth weight and low birth weight: $108(5 \%)$

${ }^{\mathrm{b}}$ Among live deliveries

${ }^{\mathrm{c}}$ Among live children

and in cord blood. Thyroid function (thyroid stimulating hormone, free and total thyroxine, free and total triiodothyronine, and thyroid peroxidase antibody) is being measured in serum samples collected at $<18$ weeks, and maternal sex hormones (fractionated estrogens, free and total testosterone, estradiol, estrone, estriol, sex hormone binding globulin, and dehydroepiandrosterone) are being measured in the third pregnancy interval. Genome-wide methylome and transcriptome analyses will be performed on cord blood samples. 
Table 5 Questionnaire and prenatal/neonatal specimen availability by time point, live deliveries $(\mathrm{n}=2000)$

\begin{tabular}{|c|c|c|c|c|c|}
\hline & \multicolumn{3}{|c|}{ During pregnancy gestational age, weeks } & \multirow{2}{*}{$\begin{array}{l}\text { Any time during } \\
\text { pregnancy }\end{array}$} & \multirow[t]{2}{*}{ At birth } \\
\hline & $<18$ & $18-25$ & $>25$ & & \\
\hline Questionnaires, n (\%) & $2000(100 \%)$ & $1726(86 \%)$ & & $2000(100 \%)$ & $1080(54 \%)$ \\
\hline Diet History Questionnaire II, n (\%) & & $1406(70 \%)$ & & $1406(70 \%)$ & \\
\hline Maternal urine, n (\%) & $1777(89 \%)$ & $1318(66 \%)$ & $1627(81 \%)$ & $1938(97 \%)$ & \\
\hline Maternal saliva, n (\%) & $1603(80 \%)$ & $1074(54 \%)$ & $1458(73 \%)$ & $1868(93 \%)$ & \\
\hline Maternal whole blood, n (\%) & $1146(57 \%)$ & $479(24 \%)$ & $850(43 \%)$ & $1514(76 \%)$ & \\
\hline Maternal PAXgene RNA tube, n (\%) & $1174(59 \%)$ & & $850(43 \%)$ & $1441(72 \%)$ & \\
\hline Maternal plasma, n (\%) & & $477(24 \%)$ & & $477(24 \%)$ & \\
\hline Maternal serum, n (\%) & $1155(58 \%)$ & $492(25 \%)$ & $858(43 \%)$ & $1521(76 \%)$ & \\
\hline Maternal vaginal swab, n (\%) & $1088(54 \%)$ & $860(43 \%)$ & $1173(59 \%)$ & $1491(75 \%)$ & \\
\hline Placental core, n (\%) & & & & & $1509(75 \%)$ \\
\hline Umbilical cord, $\mathrm{n}(\%)$ & & & & & $1491(75 \%)$ \\
\hline Cord blood (PAXgene RNA tube), n (\%) & & & & & $1392(70 \%)$ \\
\hline Cord blood (Whole), n (\%) & & & & & $1059(53 \%)$ \\
\hline Cord serum, n (\%) & & & & & $886(44 \%)$ \\
\hline Cold plasma, n (\%) & & & & & $417(21 \%)$ \\
\hline Neonatal meconium, $\mathrm{n}(\%)$ & & & & & $396(20 \%)$ \\
\hline
\end{tabular}

\section{Data collection in the postnatal phase}

ECHO also supports the postnatal phase of the study through age 2 years with in-person assessments that occur at ages 12-23 and 24-35 months outside of regular medical care. Each visit is coupled with a comprehensive questionnaire filled out by mothers prior to or during the visit. In addition, evaluations include questionnaires administered online or via phone at 4-7, 8-11, and 18-23 months (Table 6). Mothers are encouraged to use the information from visits with their primary care providers to report on children's anthropometric measures in every questionnaire. They also report on history of hospitalizations and illnesses. Questionnaires evaluate breastfeeding and infant feeding history, sleep patterns, childcare, and media exposures. Child early development is assessed using the Ages and Stages Questionnaire-Third Edition (ASQ-3) [46] and the World Health Organization questionnaire on achievement of major gross motor milestones [47]. Parental rating of the child's behavior is assessed at 12-17 months using the Brief Infant-Toddler Social and Emotional Assessment (BITSEA) [48], and at 24-35 months using the Child Behavior Checklist (CBCL) [49]. Children's language development is assessed using the Language Development Survey (LDS) at age 24-35 months [49]. When the children are 24-35 months, we obtain information on parenting using the Parenting Scale [50] and family relationship using the Family Environment Scale (FES) [51].

In-person visits at ages $12-23$ and $24-35$ months take approximately $60 \mathrm{~min}$ and include collection of child urine samples and anthropometric measures; a dual-energy
X-ray absorptiometry scan to evaluate bone, fat and muscle mass; and measurement of anogenital distance, a wellknown proxy for sex steroid exposure in utero [52]. With ECHO support, 12-23 and 24-35 month urine samples will be assayed for the same analytes as the prenatal samples. During these in-person visits, we also ask mothers to complete a 24-hour recall of their child's food intake using the Automated Self-Administered 24-hour Dietary Assessment Tool (ASA-24). Maternal depression is assessed via the Edinburgh Postnatal Depression Scale (EPDS) in the 4-7 month questionnaire and by the Brief Symptoms Inventory (BSI) in the 18-23 month questionnaire [53, 54]. At age 24-35 months, maternal stress is assessed using the Perceived Stress Scale (PSS) [55]. Food insecurity is assessed at 4-7 months using the US Department of Agriculture's Core Food Insecurity Module.

Parents also consent to prospective, passive data collection from the electronic health record for pediatric visits. This includes anthropometric measurements; outpatient medical diagnoses; medications/immunizations; laboratory values including hemoglobin and blood lead; and inpatient and emergency room visit records, including diagnoses, procedures, and medications.

Because NYU CHES is a participating cohort in the NIH ECHO Program, our data collection is being aligned and supplemented, as necessary, to fulfill the ECHO-wide Cohort Data Collection Protocol (EWCP). Harmonized data will then be consolidated across the $70 \mathrm{ECHO}$ cohorts nationally, comprising a sample of at least 50,000 children. This will enhance the statistical power and generalizability of findings related to environmental and preventable 
Table 6 Variables measured in NYU CHES through child age 2 years

\begin{tabular}{|c|c|c|c|c|c|c|c|c|c|}
\hline & $<18$ weeks & $18-25$ weeks & $>25$ weeks & Birth & 4-7 months & $8-11$ months & $12-17$ months & $18-23$ months & 24-35 months \\
\hline \multicolumn{10}{|c|}{ Maternal/family characteristics } \\
\hline $\begin{array}{l}\text { Family sociodemo- } \\
\text { graphic factors }\end{array}$ & Q, EHR & EHR & & & Q & Q & Q & Q & Q \\
\hline $\begin{array}{l}\text { Gestational hyperten- } \\
\text { sion, preeclampsia } \\
\text { and gestational } \\
\text { diabetes }\end{array}$ & Q, EHR & Q, EHR & EHR & Q & Q & & & & \\
\hline $\begin{array}{l}\text { Current pregnancy } \\
\text { information, includ- } \\
\text { ing morning sick- } \\
\text { ness and vitamin/ } \\
\text { medication use }\end{array}$ & Q & Q & & Q & & & & & \\
\hline $\begin{array}{l}\text { Maternal stress, } \\
\text { depression, or } \\
\text { anxiety }\end{array}$ & Q & Q & Q & & Q & & & Q & Q \\
\hline $\begin{array}{l}\text { Adverse events during } \\
\text { pregnancy }\end{array}$ & EHR & EHR & EHR & Q & & & & & \\
\hline Reproductive history & Q, EHR & EHR & EHR & & & & & & \\
\hline $\begin{array}{l}\text { General health and } \\
\text { health history }\end{array}$ & Q, EHR & Q, EHR & & Q, EHR & & & & Q & Q \\
\hline Sleep & Q & Q & & Q & & & & & \\
\hline Physical activity & Q & Q & Q & Q & & & & & \\
\hline $\begin{array}{l}\text { Maternal substance } \\
\text { use }\end{array}$ & Q & Q & & Q & Q & Q & Q & Q & Q \\
\hline $\begin{array}{l}\text { Housing character- } \\
\text { istics }\end{array}$ & Q & Q & & Q & & & & Q & \\
\hline Home environment & & & & & & & & Q & \\
\hline Pets and pests & Q & Q & & Q & Q & Q & Q & Q & \\
\hline $\begin{array}{l}\text { Maternal social sup- } \\
\text { port, exposure to } \\
\text { domestic violence }\end{array}$ & & Q & & & & & & Q & Q \\
\hline Diet & & Q & & & & & & & \\
\hline $\begin{array}{l}\text { Labor and delivery of } \\
\text { previous birth, and } \\
\text { previous pregnan- } \\
\text { cies }\end{array}$ & Q, EHR & & & & Q, EHR & & & & \\
\hline $\begin{array}{c}\text { Maternity leave/ } \\
\text { employment }\end{array}$ & Q & Q & & Q & Q & & & & \\
\hline Childcare & & & & & $\mathrm{Q}$ & Q & $\mathrm{Q}$ & Q & \\
\hline Food insecurity & Q & & & & Q & & & & \\
\hline $\begin{array}{l}\text { Parenting and family } \\
\text { cohesion }\end{array}$ & & & & & & & & & Q \\
\hline \multicolumn{10}{|l|}{ Child characteristics } \\
\hline Diet and feeding & & & & & Q & Q & Q & Q & \\
\hline $\begin{array}{l}\text { General health, } \\
\text { medications, and } \\
\text { hospitalizations }\end{array}$ & & & & & Q, EHR & Q, EHR & Q, EHR & Q, EHR & Q, EHR \\
\hline Early development & & & & & Q & Q & $\mathrm{Q}$ & Q & Q \\
\hline $\begin{array}{l}\text { Language develop- } \\
\text { ment }\end{array}$ & & & & & & & & & Q \\
\hline $\begin{array}{l}\text { Emotional and behav- } \\
\text { ioral development }\end{array}$ & & & & & & & $\mathrm{Q}$ & & Q \\
\hline $\begin{array}{l}\text { Floor time and } \\
\text { devices that hold } \\
\text { the baby }\end{array}$ & & & & & $\mathrm{Q}$ & Q & Q & & \\
\hline Media exposure & & & & & Q & Q & Q & Q & Q \\
\hline Sleep & & & & & Q & Q & Q & Q & \\
\hline \multicolumn{10}{|l|}{ Clinical measures } \\
\hline $\begin{array}{l}\text { Fetal sonographic } \\
\text { measurements }\end{array}$ & Measured & Measured & Measured & & & & & & \\
\hline
\end{tabular}


Table 6 (continued)

\begin{tabular}{|c|c|c|c|c|c|c|c|c|c|}
\hline & $<18$ weeks & $18-25$ weeks & $>25$ weeks & Birth & 4-7 months & $8-11$ months & $12-17$ months & 18-23 months & 24-35 months \\
\hline $\begin{array}{l}\text { Brachial artery } \\
\text { distensibility, blood } \\
\text { pressure }\end{array}$ & & & & & & & & M:Measured & $\begin{array}{l}\text { M:Measured } \\
\text { C:Measured }\end{array}$ \\
\hline Weight and height & $\begin{array}{l}\text { M:Q, EHR } \\
P: Q\end{array}$ & M:EHR & M:EHR & $\begin{array}{l}\text { M:Q, EHR } \\
\text { C:Q, EHR }\end{array}$ & $\mathrm{C}: \mathrm{Q}$ & $\mathrm{C}: \mathrm{Q}$ & $\mathrm{C}: \mathrm{Q}$ & $\begin{array}{l}\text { M:Measured } \\
\text { C:Measured }\end{array}$ & $\begin{array}{l}\text { M:Measured } \\
\text { C:Measured }\end{array}$ \\
\hline Anogenital distance & & & & Measured & & & & Measured & Measured \\
\hline $\begin{array}{l}\text { Fat, lean mass (DXA } \\
\text { scan) }\end{array}$ & & & & & & & & C:Measured & C:Measured \\
\hline \multicolumn{10}{|l|}{ Biological measures } \\
\hline $\begin{array}{l}\text { Bisphenols, phthalate } \\
\text { metabolites, } \\
\text { dialkylphosphate } \\
\text { metabolites, } \\
\text { polycyclic aromatic } \\
\text { hydrocarbon } \\
\text { metabolites }\end{array}$ & M:Urine & M:Urine & M:Urine & & & & & C:Urine & C:Urine \\
\hline $\begin{array}{l}\text { Biomarkers of oxidant } \\
\text { stress }\end{array}$ & M:Urine & M:Urine & M:Urine & & & & & C:Urine & C:Urine \\
\hline Metabolomics & M:Urine & M:Urine & M:Urine & & & & & C:Urine & C:Urine \\
\hline $\begin{array}{l}\text { Adiponectin and } \\
\text { leptin }\end{array}$ & & & & Cord serum & & & & & \\
\hline $\begin{array}{l}\text { Thyroid function and } \\
\text { Thyroid peroxidase } \\
\text { antibody }\end{array}$ & M:Serum & & & & & & & & \\
\hline $\begin{array}{l}\text { Biomarkers of } \\
\text { inflammation } \\
\text { and endothelial } \\
\text { dysfunction }\end{array}$ & M:Serum & M:Serum & M:Serum & Cord serum & & & & & \\
\hline Sex steroids & & & M:Serum & & & & & & \\
\hline $\begin{array}{l}\text { Methylomics } \\
\text { (genome-wide) }\end{array}$ & & & & Cord PAXgene & & & & & \\
\hline $\begin{array}{l}\text { Transcriptomics } \\
\text { (genome-wide) }\end{array}$ & & & & Cord PAXgene & & & & & \\
\hline
\end{tabular}

$Q$ questionnaire as completed by mothers, $E H R$ electronic health record, $C$ child, $M$ maternal, $P$ partner

predictors of childhood disease and disability. The EWCP includes measures of neurodevelopment, asthma, obesity, pre-, peri-, and immediate postnatal outcomes, as well as positive health. To align our cohort with the EWCP and ensure its completion as children evolve through life stages beyond infancy, we have planned follow-up visits at 36-59 and $60-83$ months of age.

\section{Data management and statistical power}

\section{Data preparation}

The questionnaire data are collected and managed using REDCap electronic data capture tools hosted at NYU Langone Health [56, 57]. An extensive set of validation rules and skip logic are implemented to ensure data quality. Data collected by measurements are recorded in REDCap or downloaded from the specific softwares to a secure location on the study server. Information from participants' electronic health records is obtained from EPIC (all sites) and QuadraMed (Bellevue). The data from each data source are checked and cleaned. All missing data, implausible values, logical errors, and outliers are reviewed. Then, the data from all sources are harmonized and consolidated.

\section{Privacy protection}

The study data are securely stored on the NYU Langone Health network behind a firewall. The data are accessible exclusively to the NYU CHES staff authorized by the Principal Investigator. A set of physical, technical, and administrative controls are implemented to ensure data protection. Data access rights are assigned to personnel according to their role in the study.

The links between participants' unique identification numbers and potentially identifying and protected health information (PHI) are securely stored on the NYU Langone 
Health server and are accessible only to authorized study personnel. With participant permission, specimens will be banked indefinitely with identifying links. A data transfer agreement is required for release of any data to external investigators. Depending on the Institutional Review Board approval stipulations, the datasets for analyses are either deidentified or limited.

\section{Statistical power}

Power analyses are presented in Table 7 and summarize minimally detectable difference in the unit of standard deviation (SD) for a continuous outcome between exposed and unexposed groups according to various exposure prevalences. For example, when $10 \%$ of the cohort are the exposured, the NYU CHES full cohort with the targeted 2000 live births will have $80 \%$ power to detect a difference as small as 0.209 SD between the exposure groups at a type I error level of 5\% using a two-sample $t$ test. Considering the exposure variable as continuous variable, the sample size of 2000 will have $80 \%$ power to detect a change of $0.063 \mathrm{SD}$ in the outcome for one SD increase in the exposure variable. This effect size translates into $0.13 \mathrm{BMI}$ in children, which is much smaller that the main effect previously reported for other environmental exposures such as dietary factors [58]. Note that many outcome variables will be repeatedly measured in NYU CHES, and this can lead to increased statistical power to study exposure effects. Minimally detected odds ratios for $20 \%$ exposure when the incidence in the exposed group is $5 \%$ or $10 \%$ are 1.65 and 1.96 , respectively, in the CHES full cohort $(n=2000)$, and these minimum detectable ORs will be improved to 1.34 and 1.24 for one SD increase in continuous exposure variable. For gene-environment interaction analysis, power analyses were performed assuming an additive mode of inheritance for the gene. With a type I error level of 5\% and the sample size of 2000, and the main effect of gene set at $0.20 \mathrm{SD}$ increment of outcome per SD change in the exposure and the main effect of environment at $0.05 \mathrm{SD}$ increment of outcome per SD change in the exposure, we have $80 \%$ power to detect minimal detectable interaction effect of 0.09 , if the allele frequency is $40 \%$. For allele frequencies equal to $30 \%, 20 \%$, and $10 \%$, the minimal detectable interaction effects will be $0.10,0.11$, and 0.15 , respectively.

\section{Strengths and limitations}

A leading strength of the cohort is its socioeconomic and racial/ethnic diversity, which is a byproduct of recruitment at a diverse array of clinical venues. Our cohort also includes a wide range of data from various sources-biological samples, questionnaires, and physical
Table 7 Minimally detectable difference in standard deviation between groups according to exposure prevalence

measurements from in-person visits, as well as electronic health records. We also have complete address histories for all NYU Langone participants that will allow us to geocode and use spatial mapping to assess spatially defined factors. Extant geospatial linkages include databases containing information on air pollution, noise, and neighborhood characteristics, including access to parks and healthy foods. The collection of specimens in three phases of pregnancy is another strength in that it allows us to examine trajectories of exposures over time and average them to account for variability due to non-persistence of certain chemical exposures. Our cohort is also well positioned to examine mixtures of non-persistent environmental chemicals at each time point in pregnancy and infancy. In addition, collection of infant samples is rare and permits examination of that window of vulnerability in relation to trajectories of ex utero growth.

Although we conceived of NYU CHES in the context of examining environmental exposures and child obesity, as part of the EWCP that is actively being implemented, our cohort is extremely well poised to examine neurodevelopmental and respiratory effects of prenatal and infant exposures to phthalates, bisphenols, OP pesticides, and PAHs, as well.

Selective non-response during the follow-up and retention are always challenges with an urban cohort, and the population of New York City is particularly fluid. Nonetheless, the comparison of mothers who provided consent to participate in the postnatal follow-up with those who decided not to (for reasons such as outmigration from New York City, lack of interest, or loss of contact with the study) confirms that participation in the postnatal phase of NYU CHES has not been selective. As shown in Table 8, participating mothers had similar age at enrollment, race/ethnic background, marital status, income, and employment status. Minor differences in characteristics 
Table 8 Descriptive characteristics of NYU CHES study participants by postnatal enrollment status

\begin{tabular}{|c|c|c|}
\hline \multirow[t]{2}{*}{ Characteristic $^{\mathrm{a}}$} & $\begin{array}{l}\text { Participants consented } \\
\text { to postnatal phase } \\
\end{array}$ & $\begin{array}{l}\text { Participants not } \\
\text { consented to postnatal } \\
\text { phase }\end{array}$ \\
\hline & & \\
\hline Age at enrollment, mean (SD), years & $32.0(5.5)$ & $31.2(5.7)$ \\
\hline \multicolumn{3}{|l|}{ Race/ethnicity, n (\%) } \\
\hline Hispanic & $769(49 \%)$ & $196(49 \%)$ \\
\hline White, not of Hispanic origin & $546(34 \%)$ & $131(32 \%)$ \\
\hline Black, not of Hispanic origin & $84(5 \%)$ & $30(7 \%)$ \\
\hline Asian & $145(9 \%)$ & $35(9 \%)$ \\
\hline Other & $14(1 \%)$ & $3(1 \%)$ \\
\hline Multiple & $35(2 \%)$ & $7(2 \%)$ \\
\hline \multicolumn{3}{|l|}{ Marital status, n (\%) } \\
\hline Married/living with a partner & $1410(88 \%)$ & $347(86 \%)$ \\
\hline Divorced/separated & $38(3 \%)$ & $6(2 \%)$ \\
\hline Single/widowed & $149(9 \%)$ & $49(12 \%)$ \\
\hline \multicolumn{3}{|l|}{ Education, n (\%) } \\
\hline High school or less & $491(31 \%)$ & $144(36 \%)$ \\
\hline Some college but no degree & $187(12 \%)$ & $42(11 \%)$ \\
\hline Associate degree & $76(5 \%)$ & $22(6 \%)$ \\
\hline Bachelor's degree & $360(22 \%)$ & $97(24 \%)$ \\
\hline Post graduate degree & $472(30 \%)$ & $92(23 \%)$ \\
\hline \multicolumn{3}{|l|}{ Household income, n (\%) } \\
\hline Less than $\$ 30,000$ & $258(17 \%)$ & $62(16 \%)$ \\
\hline$\$ 30,000-\$ 49,999$ & $128(8 \%)$ & $40(11 \%)$ \\
\hline$\$ 50,000-\$ 74,999$ & $104(7 \%)$ & $28(7 \%)$ \\
\hline$\$ 75,000-\$ 99,999$ & $70(4 \%)$ & $23(6 \%)$ \\
\hline$\$ 100,000$ or more & $630(40 \%)$ & $129(34 \%)$ \\
\hline Don’t know & $368(24 \%)$ & $101(26 \%)$ \\
\hline Employed, n (\%) & $1046(66 \%)$ & $260(65 \%)$ \\
\hline \multicolumn{3}{|l|}{ Insurance, $\mathrm{n}(\%)$} \\
\hline Public & $798(50 \%)$ & $221(55 \%)$ \\
\hline Private & $786(50 \%)$ & $175(45 \%)$ \\
\hline \multicolumn{3}{|l|}{$\begin{array}{l}\text { Use of cigarettes and other related products during } \\
\text { pregnancy, } \mathrm{n}(\%)\end{array}$} \\
\hline Never & $1450(91 \%)$ & $362(90 \%)$ \\
\hline Used, stopped in pregnancy & $127(8 \%)$ & $35(9 \%)$ \\
\hline Used, continued in pregnancy & $18(1 \%)$ & $5(1 \%)$ \\
\hline \multicolumn{3}{|l|}{ Alcohol use during pregnancy, n (\%) } \\
\hline Never & $518(32 \%)$ & $144(36 \%)$ \\
\hline Used, stopped in pregnancy & $809(51 \%)$ & $215(54 \%)$ \\
\hline Used, continued in pregnancy & $268(17 \%)$ & $40(10 \%)$ \\
\hline Pre-pregnancy body mass index, mean (SD), $\mathrm{kg} / \mathrm{m}^{2}$ & $26.4(5.9)$ & $25.8(5.1)$ \\
\hline Nulliparous, n (\%) & $779(49 \%)$ & $223(56 \%)$ \\
\hline
\end{tabular}

${ }^{\mathrm{a}} 1597$ mothers of 1624 children such as education (higher educated mother participated in the follow-up), alcohol use (mothers who drank during pregnancy participated in the follow-up), and parity (participation rate of nulliparous mother was lower than parous mothers) will be considered in future analysis using appropriate epidemiological methods (e.g., inverse probability weighting). 
Currently, the study collects information on fathers/partners through maternal reporting. These data provide valuable information including sociodemographic characteristics and weight and height, but the study remains limited in direct assessments of the fathers/partners as well as their evaluation of children's growth and development. We also have not collected health histories or specimen from any parents biologically related to the child other than the birth mother.

\section{Collaboration}

Regular data transmission to the NIH ECHO Data Analysis Center will permit centralized data analyses around common hypotheses, as well as preparation of de-identified and restricted datasets. For more information, see www.nih. gov/echo. For investigators interested in our primary data or in ancillary studies, NYU CHES has developed publications, specimen, and data sharing policies that are meant to encourage maximal use of this rich resource. More information can be obtained by contacting NYULHEnvPeds@ nyulangone.org.

Acknowledgements Abigail Gaylord, Adeyinka Ajayi, Adriana Garcia, Aisha Dar, Alexis Mandon, Alice Trye, Alyssa Polak, Amit Saxena, Ana Pacheco Ochoa, Ana Ramirez, Anandini Suri, Andrea Larkin Papa, Andrea Nardello, Andrew Ailoje, Anna Kheyfets, Anne Lorraine Dumadag, Ashley Jones, Benjamin Barnett, Bettina Babu, Blanca Vargas, Bo Gu, Camille McNally, Carly Batt, Carolena Rojas Marcos, Chiemika Nwosu, Christiana Harry, Christina Awada, Christopher Haggar, Daniel Wohl, Danisha Dennie, David Fenyo, Denise Mallick, Doris Descorbeth, Douglas Donnelly, Eldad Cano, Elle Wszeborowski, Emely Tejeda, Emma Cowles, Eric Mallow, Evelyn Hernandez, Felix Le Coadic, Freddy Loffredo, Giovanna Lopez, George Nikoloudakis, Ha Young Kyung, Hai Man He, Hannah Bava, Heather Jao, Ho Fei Sit, Isabella Possagnoli, James Deats, Jamie Lee Tavarez, Jasmine Raymer, Jassly Naval, Jennifer B. Lee, Jennifer Carpio, Jessica Ospina, Jill Buyon, Jocelyn Majano, Jose Lugo Eslava, Joseline M Cruz Vasquez, Juan Pablo Robayo, Julia Malits, Kasandra Cisneros, Kayla Rae Farrell, Kirtan Kaur, Kristine Karibandi, Kevin Mendoz, Lauren Burdine, Liliany Nigam, Lillian Walton Masters, Lisa Nathan, Lonny Dym, Makhethe Mpoti, Manuel Hernandez, Maria del Mar Colon, Maria Sanchez Garcia, Marina Rosado, Mary Jo Messito, Matthew Shong, Meera T, Melissa Robbins, Michael Ferro, Michelle Gorchynski, Miriam Peters, Miriam Woodward, Nader Daoud, Nathalia Schettino, Onassis Castillo Ceballo, Paridhi Bhargava, Peggy Hsieh, Pema Sherpa, Peter Izmirly, Rachel Marconi, Rakan Alqaqaa, Ranga Bharadwaj, Rifat Iqbal, Robert Clancy, Roberta Scheinmann, Rummanu Yeasin, Sara Brubaker, Sara Long, Sarah Lazaros, Sarah Watson, Yuyan Wang, Sarvani Ramcharran, Shikha Chandarana, Shilpi MehtaLee, Shivani Karthikeyan, Simran Sahansra, Stefani Yanez, Stephanie Vazquez, T Meera, Tatiana Wilson, Teresa Attina, Terri-Anne Bennet, and Yutian Mu. We thank Vice Dean for Science Dafna Bar-Sagi and the NYU School of Medicine's Office of Science and Research, as well as Drs. Catherine Manno and David Keefe.

Funding NYU CHES is supported by institutional funds of NYU Grossman School of Medicine as well as the NIH Office of the Director (UG3/UH3OD023305).
Open Access This article is licensed under a Creative Commons Attribution 4.0 International License, which permits use, sharing, adaptation, distribution and reproduction in any medium or format, as long as you give appropriate credit to the original author(s) and the source, provide a link to the Creative Commons licence, and indicate if changes were made. The images or other third party material in this article are included in the article's Creative Commons licence, unless indicated otherwise in a credit line to the material. If material is not included in the article's Creative Commons licence and your intended use is not permitted by statutory regulation or exceeds the permitted use, you will need to obtain permission directly from the copyright holder. To view a copy of this licence, visit http://creativecommons.org/licenses/by/4.0/.

\section{References}

1. National Research Council. Pesticides in the diets of infants and children. Washington: National Academies Press; 1993.

2. Barker DJP, Osmond C, Forsen TJ, Kajantie E, Eriksson JG. Trajectories of growth among children who have coronary events as adults. N Engl J Med. 2005;353(17):1802.

3. Barker DJP, Godfrey KM, Osmond C, Bull A. The relation of fetal length, ponderal index and head circumference to blood pressure and the risk of hypertension in adult life. Paediatr Perinat Epidemiol. 1992;6(1):35-44. https://doi. org/10.1111/j.1365-3016.1992.tb00741.x.

4. Barker DJ. The fetal and infant origins of adult disease. BMJ. 1990;301(6761):1111.

5. Barker DJ, Osmond C. Infant mortality, childhood nutrition, and ischaemic heart disease in England and Wales. Lancet. 1986;1(8489):1077-81.

6. Trasande L, Cronk C, Durkin M, et al. Environment, obesity and the National Children's Study. Environ Health Perspect. 2009;117(2):159-66. https://doi.org/10.1289/ehp.11839.

7. Tylavsky FA, Ferrara A, Catellier DJ, et al. Understanding childhood obesity in the US: the NIH environmental influences on child health outcomes (ECHO) program. Int J Obes (Lond). 2019. https://doi.org/10.1038/s41366-019-0470-5.

8. Demerath EW, Li J, Sun SS, et al. Fifty-year trends in serial body mass index during adolescence in girls: the Fels Longitudinal Study. Am J Clin Nutr. 2004;80(2):441.

9. Strauss RS, Knight J. Influence of the home environment on the development of obesity in children. Am Acad Pediatr. 1999; 103:e85.

10. Berkey CS, Rockett HRH, Field AE, et al. Activity, dietary intake, and weight changes in a longitudinal study of preadolescent and adolescent boys and girls. Am Acad Pediatr. 2000;105:E56.

11. Siervogel RM, Wisemandle W, Maynard LM, Guo SS, Chumlea WC, Towne B. Lifetime overweight status in relation to serial changes in body composition and risk factors for cardiovascular disease: the Fels Longitudinal Study. NAASO. 2000;8:422-30.

12. Guo SS, Wu W, Chumlea WC, Roche AF. Predicting overweight and obesity in adulthood from body mass index values in childhood and adolescence. Am J Clin Nutr. 2002;76(3):653-8.

13. Parsons TJ. Fetal and early life growth and body mass index from birth to early adulthood in 1958 British cohort: longitudinal study. BMJ. 2001;323(7325):1331-5.

14. Lake JK, Power C, Cole TJ. Child to adult body mass index in the 1958 British birth cohort: associations with parental obesity. Arch Dis Child. 1997;77(5):376.

15. Freedman DS, Khan LK, Serdula MK, Dietz WH, Srinivasan SR, Berenson GS. The relation of childhood BMI 
to adult adiposity: the Bogalusa Heart Study. Pediatrics. 2005;115(1):22-7.

16. Lauer RM, Clarke WR, Burns TL. Obesity in childhood: the Muscatine Study. Zhonghua Min Guo Xiao Er Ke Yi Xue Hui Za Zhi. 1997;38(6):432-7.

17. Moll PP, Burns TL, Lauer RM. The genetic and environmental sources of body mass index variability: the Muscatine Ponderosity Family Study. Am J Hum Genet. 1991;49:s1243-50.

18. Nelson MC, Gordon-Larsen P, Song Y, Popkin BM. Built and social environments associations with adolescent overweight and activity. Am J Prev Med. 2006;31(2):109-17.

19. Gordon-Larsen P, Nelson MC, Page P, Popkin BM. Inequality in the built environment underlies key health disparities in physical activity and obesity. Pediatrics. 2006;117(2):417-24.

20. Thompson DR, Obarzanek E, Franko DL, et al. Childhood overweight and cardiovascular disease risk factors: the national heart, lung, and blood institute growth and health study. J Pediatr. 2007;150(1):18-25.

21. Nader PR, O'Brien M, Houts R, et al. Identifying risk for obesity in early childhood. Pediatrics. 2006;118(3):e594.

22. Ben-Shlomo Y, Kuh D. A life course approach to chronic disease epidemiology: conceptual models, empirical challenges and interdisciplinary perspectives. IEA. 2002;31:285-93.

23. Freedman DS, Khan LK, Serdula MK, Ogden CL, Dietz WH. Racial and ethnic differences in secular trends for childhood BMI, weight, and height. Obesity (Silver Spring). 2006;14(2):301-8.

24. Strauss RS, Pollack HA. Epidemic increase in childhood overweight, 1986-1998. JAMA. 2001;286(22):2845-8.

25. Kipke MD, Iverson E, Moore D, et al. Food and park environments: neighborhood-level risks for childhood obesity in East Los Angeles. J Adolesc Health. 2007;40(4):325-33.

26. Heindel JJ, Newbold R, Schug TT. Endocrine disruptors and obesity. Nat Rev Endocrinol. 2015;11(11):653-61. https://doi. org/10.1038/nrendo.2015.163.

27. Desvergne B, Feige JN, Casals-Casas C. PPAR-mediated activity of phthalates: a link to the obesity epidemic? Mol Cell Endocrinol. 2009;304(1-2):43-8. https://doi.org/10.1016/j.mce.2009.02.017.

28. Howdeshell KL, Hotchkiss AK, Thayer KA, Vandenbergh JG, vom Saal FS. Exposure to bisphenol A advances puberty. Nature. 1999;401(6755):763-4.

29. Masuno H, Kidani T, Sekiya K, et al. Bisphenol A in combination with insulin can accelerate the conversion of 3T3-L1 fibroblasts to adipocytes. J Lipid Res. 2002;43(5):676-84.

30. Sun Q, Yue P, Deiuliis J, et al. Ambient air pollution exaggerates adipose inflammation and insulin resistance in a mouse model of diet-induced obesity. Circulation. 2009;119:538-46.

31. Zheng Z, Xu X, Zhang X, et al. Exposure to ambient particulate matter induces a NASH-like phenotype and impairs hepatic glucose metabolism in an animal model. J Hepatol. 2013;58(1):14854. https://doi.org/10.1016/j.jhep.2012.08.009.

32. Slotkin TA. Does early-life exposure to organophosphate insecticides lead to prediabetes and obesity? Reprod Toxicol. 2011;31(3):297-301. https://doi.org/10.1016/j.repro tox.2010.07.012.

33. Braun JM, Lanphear BP, Calafat AM, et al. Early-life bisphenol a exposure and child body mass index: a prospective cohort study. Environ Health Perspect. 2014;122(11):1239-45. https:// doi.org/10.1289/ehp.1408258.

34. Harley KG, Aguilar Schall R, Chevrier J, et al. Prenatal and postnatal bisphenol A exposure and body mass index in childhood in the CHAMACOS cohort. Environ Health Perspect. 2013;121(4):514-20, 20e1-6. https://doi.org/10.1289/ehp.12055 48.

35. Valvi D, Casas M, Mendez MA, et al. Prenatal bisphenol a urine concentrations and early rapid growth and overweight risk in the offspring. Epidemiology (Cambridge, MA). 2013;24(6):791-9. https://doi.org/10.1097/ede.0b013e3182a67822.

36. Valvi D, Casas M, Romaguera D, et al. Prenatal phthalate exposure and childhood growth and blood pressure: evidence from the Spanish INMA-Sabadell Birth Cohort Study. Environ Health Perspect. 2015. https://doi.org/10.1289/ehp.1408887.

37. Maresca MM, Hoepner LA, Hassoun A, et al. Prenatal exposure to phthalates and childhood body size in an urban cohort. Environ Health Perspect. 2015. https://doi.org/10.1289/ehp.1408750.

38. Hygiene. NYCDoHaM. Epiquery: NYC interactive health data system: vital statistics birth data. http://nyc.gov/health/epiquery. Accessed 12 Apr 2019.

39. Lu M, Halfon N. Racial and ethnic disparities in birth outcomes: a life-course perspective. Matern Child Health J. 2003;7(1):13-30. https://doi.org/10.1023/A:1022537516969.

40. Oberg CN, Rinaldi M. Pediatric health disparities. Curr Probl Pediatr Adolesc Health Care. 2006;36(7):251-68.

41. Rini CK, Dunkel-Schetter C, Wadhwa PD, Sandman CA. Psychological adaptation and birth outcomes: the role of personal resources, stress, and sociocultural context in pregnancy. Health Psychol. 1999;18(4):333-45.

42. Gilbody S, Richards D, Brealey S, Hewitt C. Screening for depression in medical settings with the Patient Health Questionnaire (PHQ): a diagnostic meta-analysis. J Gen Intern Med. 2007;22(11):1596-602. https://doi.org/10.1007/s1160 6-007-0333-y.

43. Buysse DJ, Reynolds CF 3rd, Monk TH, Berman SR, Kupfer DJ. The Pittsburgh Sleep Quality Index: a new instrument for psychiatric practice and research. Psych Res. 1989;28(2):193-213.

44. Subar AF, Thompson FE, Kipnis V, et al. Comparative validation of the Block, Willett, and National Cancer Institute food frequency questionnaires: the Eating at America's Table Study. Am J Epidemiol. 2001;154(12):1089-99.

45. Craig CL, Marshall AL, Sjöström M, et al. International physical activity questionnaire: 12 -country reliability and validity. Med Sci Sports Exerc. 2003;35(8):1381-95.

46. Squires J, Bricker D. Ages \& Stages Questionnaires [R], (ASQ-3 $[\mathrm{TM}])$ : a parent-completed child-monitoring system. Baltimore: Brookes Publishing Company; 2009.

47. WHO Multicentre Growth Reference Study Group. WHO Motor Development Study: windows of achievement for six gross motor development milestones. Acta Paediatr Suppl. 2006;450:86-95.

48. Briggs-Gowan MJ, Carter AS, Irwin JR, Wachtel K, Cicchetti DV. The Brief Infant-Toddler Social and Emotional Assessment: screening for social-emotional problems and delays in competence. J Pediatr Psychol. 2004;29(2):143-55.

49. Achenbach TM, Rescorla LA. Manual for ASEBA preschool forms \& profiles. Burlington: University of Vermont, Research Center for Children, Youth, \& Families; 2000.

50. Arnold DS, O'Leary SG, Wolff LS, Acker MM. The parenting scale: a measure of dysfunctional parenting in discipline situations. Psychol Assess. 1993;5(2):137-44. https://doi. org/10.1037/1040-3590.5.2.137.

51. Roosa MW, Beals J. Measurement issues in family assessment: the case of the family environment scale. Fam Process. 1990;29(2):191-8. https://doi.org/10.111 1/j.1545-5300.1990.00191.x.

52. Swan SH, Kristensen DMJHR. Anogenital distance: a marker of steroidal endocrine disruption. Audrology. 2018;30(4):963b72.

53. Derogatis LR. The Brief Symptom Inventory (BSI): administration, scoring, and procedures manual. 3rd ed. Minneapolis: National Computer System Inc; 1993.

54. Cox JL, Holden JM, Sagovsky R. Detection of postnatal depression: development of the 10-item Edinburgh Postnatal Depression Scale. Br J Psych. 1987;150(6):782-6. 
55. Cohen S, Kamarck T, Mermelstein R. A global measure of perceived stress. J Health Soc Behav. 1983;24(4):385-96.

56. Harris PA, Taylor R, Minor BL, et al. The REDCap consortium: building an international community of software platform partners. J Biomed Inform. 2019;95:103208. https://doi.org/10.1016/j. jbi.2019.103208.

57. Harris PA, Taylor R, Thielke R, Payne J, Gonzalez N, Conde JG. Research electronic data capture (REDCap) - a metadata-driven methodology and workflow process for providing translational research informatics support. J Biomed Inform. 2009;42(2):37781. https://doi.org/10.1016/j.jbi.2008.08.010.
58. Young AI, Wauthier F, Donnelly P. Multiple novel gene-by-environment interactions modify the effect of FTO variants on body mass index. Nat Commun. 2016;7:12724. https://doi.org/10.1038/ ncomms12724.

Publisher's Note Springer Nature remains neutral with regard to jurisdictional claims in published maps and institutional affiliations. 